\title{
Genome-Wide Screening of Cytogenetic Abnormalities in Multiple Myeloma Patients Using Array-CGH Technique: A Czech Multicenter Experience
}

\author{
Jan Smetana, ${ }^{1,2}$ Jan Frohlich, ${ }^{1,2}$ Romana Zaoralova, ${ }^{2}$ Vladimira Vallova, \\ Henrieta Greslikova, ${ }^{2}$ Renata Kupska, ${ }^{2}$ Pavel Nemec, ${ }^{1,2}$ Aneta Mikulasova, ${ }^{1,2}$ \\ Martina Almasi, ${ }^{2,3}$ Ludek Pour, ${ }^{4}$ Zdenek Adam, ${ }^{4}$ Viera Sandecka, ${ }^{4}$ Lenka Zahradová, ${ }^{5}$ \\ Roman Hajek, ${ }^{2,3,5}$ and Petr Kuglik ${ }^{1}$ \\ ${ }^{1}$ Department of Experimental Biology, Faculty of Science, Masaryk University Brno, Bohunice, 62500 Brno, Czech Republic \\ ${ }^{2}$ Babak Myeloma Group, Department of Pathological Physiology, Faculty of Medicine, Masaryk University Brno, Bohunice, \\ 62500 Brno, Czech Republic \\ ${ }^{3}$ Department of Internal Hematooncology, University Hospital Brno, Bohunice, 62500 Brno, Czech Republic \\ ${ }^{4}$ Department of Clinical Hematology, University Hospital Brno, Bohunice, 62500 Brno, Czech Republic \\ ${ }^{5}$ Faculty of Medicine, University of Ostrava and University Hospital Ostrava, Poruba, 70852 Ostrava, Czech Republic
}

Correspondence should be addressed to Petr Kuglik; kugl@sci.muni.cz

Received 20 February 2014; Accepted 2 May 2014; Published 2 June 2014

Academic Editor: Dong Soon Lee

Copyright (C) 2014 Jan Smetana et al. This is an open access article distributed under the Creative Commons Attribution License, which permits unrestricted use, distribution, and reproduction in any medium, provided the original work is properly cited.

\begin{abstract}
Characteristic recurrent copy number aberrations (CNAs) play a key role in multiple myeloma (MM) pathogenesis and have important prognostic significance for MM patients. Array-based comparative genomic hybridization (aCGH) provides a powerful tool for genome-wide classification of CNAs and thus should be implemented into MM routine diagnostics. We demonstrate the possibility of effective utilization of oligonucleotide-based aCGH in $91 \mathrm{MM}$ patients. Chromosomal aberrations associated with effect on the prognosis of MM were initially evaluated by I-FISH and were found in $93.4 \%$ (85/91). Incidence of hyperdiploidy was 49.5\% (45/91); del(13)(q14) was detected in 57.1\% (52/91); gain(1)(q21) occurred in 58.2\% (53/91); del(17)(p13) was observed in $15.4 \%$ (14/91); and $\mathrm{t}(4 ; 14)$ (p16;q32) was found in 18.6\% (16/86). Genome-wide screening using Agilent $44 \mathrm{~K}$ aCGH microarrays revealed copy number alterations in $100 \%$ (91/91). Most common deletions were found at 13q (58.9\%), $1 p(39.6 \%)$, and $8 p(31.1 \%)$, whereas gain of whole lq was the most often duplicated region (50.6\%). Furthermore, frequent homozygous deletions of genes playing important role in myeloma biology such as TRAF3, BIRC1/BIRC2, RB1, or CDKN2C were observed. Taken together, we demonstrated the utilization of aCGH technique in clinical diagnostics as powerful tool for identification of unbalanced genomic abnormalities with prognostic significance for MM patients.
\end{abstract}

\section{Introduction}

Multiple myeloma (MM) is a tumor of postgerminal center isotype switched plasma cells (PCs), which are poorly proliferative but accumulate in the bone marrow leading to anemia, hypercalcemia, and lytic bone disease. Evaluation of genetic lesions associated with prognosis of MM patients is one of the most important diagnostic tools in the field [1]. Detection of chromosomal aberrations by means of standard karyotyping is limited (about $30 \%$ of cases) due to resolution and low proliferation activity of PCs [2]. This limitation can be overcome by newer techniques, such as fluorescent in situ hybridization (FISH) with detection rate of chromosomal aberrations (CHAs) reaching over $90 \%$ of 
all cases [3]. However, this technique detects only a limited number of specific target sequences and thus provides a very limited view of the genome.

Karyotyping and FISH technique have shown that there are two major genetic subtypes in MM. Hyperdiploid MM (H-MM) is characterized by gains of odd-numbered chromosomes (e.g., chromosomes 3, 5, 7, 9, 11, 15, 19, and 21) and low incidence of $\mathrm{IgH}$ translocations and it is associated with better prognosis, whereas nonhyperdiploid MM (NH-M) is connected with worse prognosis due to frequent incidence of IgH translocations [4]. Several studies described prognostic significance of specific recurrent chromosomal aberrations, such as $\operatorname{del}(13)(\mathrm{q} 14) /$ loss of chromosome 13, $\operatorname{del}(17)(\mathrm{p} 13)$, gain(1)(q21), and $\operatorname{IgH}$ translocations for MM patients [5, 6]. However, current understanding of MM pathogenesis together with development of modern genome-wide screening techniques proves that common prognostic FISH panels are insufficient for description of genome heterogeneity of malignant PCs [7].

Complete analysis of the MM tumor genome by microarray techniques revealed novel recurrent copy number aberrations, such as deletions in $1 \mathrm{p}, 6 \mathrm{q}, 8 \mathrm{p}, 12 \mathrm{q}$, and 16q, which are now considered additional prognostic factors to highrisk features. In addition, deletions of genes involved in regulation of the NF- $\kappa \mathrm{B}$ pathway (CYLD, TRAF3, BIRC2, and $B I R C 3)$, cell cycle (CDKN2C, CDKN2A, and CDKN2B), or induction of apoptosis ( $W W O X$ and FAF1) were furthermore described by genome-wide approaches and add important information about genetic changes in MM pathogenesis [8, 9]. Recently, next-generation sequencing (NGS) techniques discovered mutations in several key genes associated with cancerogenesis, such as $K$-Ras, N-Ras, or sarcoma viral oncogene homolog B1 (BRAF) and postulated a new theory of clonal evolution of MM disease [10].

In our previous studies we showed that incidence of specific cytogenetic abnormalities, such as gain(1)(q21), del(17)(p13), and $t(4 ; 14)(\mathrm{p} 16 ; \mathrm{q} 32)$, detected by FISH in MM patients is connected with shorter overall survival for both newly diagnosed and relapsed MM patients [11, 12]. In this study, we analyzed the genomic profiles of $91 \mathrm{MM}$ samples using oligonucleotide-based aCGH technique. We focused on detailed characterization of CNAs with known prognostic importance as well as CNAs connected with $\mathrm{IgH}$ translocations and incidence of homozygous deletions in our cohort of patients in terms of better understanding and subclassification of genetic heterogeneity of MM. In addition, we performed validation analysis of the FISH panel routinely used in MM diagnostic [hyperdiploidy, del(13)(q14), $\operatorname{del}(17)(\mathrm{p} 13)$, and gain(1)(q21)] with genome-wide approach to verify the possibility of replacing the standard I-FISH technique due to scalability of genomic profiling.

\section{Material and Methods}

2.1. Patients' Characteristics. The bone marrow aspirates from 91 (46 newly diagnosed and 45 relapsed) MM patients were obtained from MM patients between 2007 and 2010 from various centers in Slovakia and the Czech Republic. Patients' clinical features are summarized in Table 1. All patients were included into this study only after they signed the informed consent form approved by the ethical committee of the hospital. The enriched samples of PCs were obtained by either anti-CD138+ immunomagnetic beads (AutoMACS Pro, Miltenyi Biotec GmbH, Bergisch Gladbach, Germany) or by fluorescent-activated cells sorting (FACS Aria, BD Biosciences, San Jose, CA, USA). Detailed protocol of sorting algorithm used in our center was described elsewhere [13]. Briefly, cutoff level of $5 \%$ for CD138+ PCs infiltration in the bone marrow was established, and sorting technique ( $<5 \%$ FACS, $>5 \%$ MACS, resp.) was used according to the manufacturer's instructions.

2.2. Microarray Analysis. Genomic DNA (gDNA) for aCGH experiments was extracted using commercially available kit (Puregene Core Kit A, Qiagen) according to manufacturer's protocol. Quality control of gDNA, digestion, labeling, and hybridization steps were performed as previously described [14]. Briefly, $1.0 \mu \mathrm{g}$ of tumor and reference DNA were independently digested with Alul and Rsal (Promega, Madison, WI, USA) for 2 hours at 37C. Agilent Euro Female/Male was used as the normal reference in the hybridization experiments. Fluorescent labeling was made by BioPrime Total for Agilent Labeling Module (Invitrogen, Carlsbad, CA, USA) with specific fluorescent dyes Alexa3 for reference and Alexa5 for tumor DNA. Labeled reactions were cleaned up and hybridized at $65 \mathrm{C}$ for 24 hours. Human Genome $4 \times 44 \mathrm{k}$ CGH Microarrays were scanned by Agilent Surescan C scanner with $5 \mu \mathrm{m}$ resolution; features were extracted with Feature Extraction software and $\log 2$ ratio data were imported and analyzed by Agilent Genomic Workbench 7.0.1.4 (Agilent Technologies, Santa Clara, CA, USA). Aberration calling was made by ADM-2 algorithm [15]. Positive aberration calls were defined by $\geq 3$ consecutive probes and overreaching 0.2 -fold change of $\log _{2}$ space. We used recommended default threshold 6 for ADM-2 algorithm with accuracy of aberration call confirmed on the basis of known FISH aberrations. The regions with detected CNAs were manually examined due to exclusion of copy number variant $(\mathrm{CNV})$ regions and only copy number changes in exons and microRNA regions were included into further analyses. To identify and eliminate common CNVs from the study, we used default Database of Genomic Variants (http://www.openhelix.com/) for hg18. Physiological loci of the $I g H, I g L-k$, and $I g L-l$ were also excluded from the analyses. Microarray data are available in the ArrayExpress database (http://www.ebi.ac.uk/arrayexpress/) under accession number E-MTAB-1792.

2.3. I-FISH Analysis. The cohort of $91 \mathrm{MM}$ patients was examined for incidence of $\mathrm{IgH}$ translocations; furthermore, the occurrence of $\operatorname{del}(13)(\mathrm{q} 14), \operatorname{del}(17)(\mathrm{p} 13)$, gain(1)(q21), and hyperdiploidy was compared with aCGH analysis in order to verify the results of whole-genome screening. The detection of PCs in the bone marrow samples was performed by 
TABLE 1: Patients' baseline characteristics.

\begin{tabular}{|c|c|}
\hline \multicolumn{2}{|l|}{ Sex } \\
\hline Males & 41 \\
\hline Females & 50 \\
\hline \multicolumn{2}{|l|}{ Age median } \\
\hline (at the time of therapy, years); range & $69(38-87)$ \\
\hline \multicolumn{2}{|l|}{ Follow-up median } \\
\hline (from therapy, months); range & $37.8(1.7-269.8)$ \\
\hline \multicolumn{2}{|l|}{ Durie-Salmon stage (from therapy) } \\
\hline I & $9(9.9 \%)$ \\
\hline II & $11(12.1 \%)$ \\
\hline III & $71(78.0 \%)$ \\
\hline \multicolumn{2}{|l|}{ Stages A-B (from therapy) } \\
\hline A & $69(75.8 \%)$ \\
\hline $\mathrm{B}$ & $22(24.2 \%)$ \\
\hline \multicolumn{2}{|l|}{ ISS stage (from therapy) } \\
\hline 1 & $22(24.2 \%)$ \\
\hline 2 & $25(27.5 \%)$ \\
\hline 3 & $44(48.4 \%)$ \\
\hline \multicolumn{2}{|l|}{ Ig isotype } \\
\hline $\operatorname{IgG}$ & $58(63.7 \%)$ \\
\hline $\operatorname{Ig} \mathrm{A}$ & $15(16.5 \%)$ \\
\hline $\operatorname{Ig} \mathrm{D}$ & $4(4.4 \%)$ \\
\hline $\operatorname{IgM}$ & $1(1.1 \%)$ \\
\hline LC only & $13(14.3 \%)$ \\
\hline \multicolumn{2}{|l|}{ Light chains } \\
\hline Kappa & $53(58.2 \%)$ \\
\hline Lambda & $38(41.8 \%)$ \\
\hline \multicolumn{2}{|l|}{ Number of previous treatment lines } \\
\hline None (first line treatment) & $46(50.6 \%)$ \\
\hline Two & $20(22.0 \%)$ \\
\hline More $(>2)$ & $25(27.4 \%)$ \\
\hline \multicolumn{2}{|l|}{ Biochemical parameter (median; min-max) } \\
\hline Haemoglobin $(\mathrm{g} / \mathrm{L})$ & $103.50(66-144)$ \\
\hline Thrombocytes (count $\times 109$ ) & $197.50(27-416)$ \\
\hline Calcium (mmol/L) & $2.32(1.47-3.64)$ \\
\hline Albumin (g/L) & $38.95(21.1-54.1)$ \\
\hline Creatinine (umol/L) & $113.00(54-1136)$ \\
\hline$\beta 2-$ Microglobulin $(\mathrm{mg} / \mathrm{L})$ & $5.18(1.8-42.16)$ \\
\hline Lactate dehydrogenase (ukat/L) & $3.80(1.52-22.92)$ \\
\hline C-reactive protein $(\mathrm{mg} / \mathrm{L})$ & $4.20(0-174)$ \\
\hline Plasma cell infiltration of bone marrow (\%) & $39.4(0.80-94.60)$ \\
\hline
\end{tabular}

immunofluorescent labeling of cytoplasmic light chain (cIgFISH), as previously reported [16], or we used CD138+ PCs obtained by cell sorting techniques. The following FISH panel of commercial DNA probes was used for analysis: LSI IGH/FGFR3 dual color probe, LSI 13q14 (RB1) spectrum green probe, LSI p53 (17p13.1) spectrum orange probe, and LSI D5S23/D5S721, CEP 9, and CEP 15 multicolor probe panel (Abbott Laboratories, Abbott Park, IL, USA). Hyperdiploidy was defined as gain of at least two of three evaluated chromosomes in a single cell. Gain(1)(q21) was assessed by homemade probe using fluorescent labeled bacterial artificial chromosome (BAC) (clone RP11-205M9); protocols for BAC isolation and labeling were followed from online resources of University of Bari, Italy (http://www.uniba.it/). Slide preparation and FISH analyses were performed according to manufacturer's protocols. We used cutoff values recommended by the European Myeloma Network [17], 20\% cutoff for deletions and numerical aberrations and $10 \%$ cutoff for translocations and $\mathrm{IgH}$ rearrangements. At least, 100 cells were scored in each sample. Digital image analysis was assessed by fluorescent microscope Olympus BX-61 equipped with a CCD Camera Vosskuhler 1300D and Lucia KARYO/FISH/CGH imaging system (Laboratory Imaging s. r. o., Prague, Czech Republic).

\section{Results}

3.1. Summary of Chromosomal Aberrations in 91 MM Patients Detected by I-FISH Technique. The evaluation of unbalanced chromosomal abnormalities (hyperdiploidy, deletion of $R B 1$, deletion of TP53, and gain/amplification 1q21) and $\mathrm{IgH}$ translocation $\mathrm{t}(4 ; 14)(\mathrm{p} 16 ; \mathrm{q} 32)$ by FISH was performed in $91 \mathrm{MM}$ patients. In our cohort, chromosomal abnormalities were detected in $93.4 \%$ (85/91) of cases. Hyperdiploidy was found in $49.5 \%$ (45/91); $\operatorname{del}(13)$ (q14) was detected in $57.1 \%$ (52/91); del(17)(p13) was observed in $15.4 \%(14 / 91)$ and gain(1)(q21) occurred in 58.2\% (53/91) of samples. The $\mathrm{t}(4 ; 14)(\mathrm{p} 16 ; \mathrm{q} 32)$ was found in $18.6 \%(16 / 86) ; 5$ cases were discarded because of low amount of evaluated cells. Incidence of translocation $\mathrm{t}(4 ; 14)(\mathrm{p} 16 ; \mathrm{q} 32)$ was associated with nonhyperdiploid ( $P=0.005)$ cases as well as gain(1)(q21) and deletion of $R B 1$ ( $P=0.025 ; P=0.052$, resp.) and corresponded with simultaneous incidence of $\operatorname{del}(13)(q 14)$ and gain $(1)(\mathrm{q} 21)(P=0.0047)$.

3.2. Results from Whole-Genome Screening of a Cohort of 91 MM Patients Using Agilent $4 \times 44 k$ Microarrays. The DNA samples from $91 \mathrm{MM}$ patients were analyzed by high-density oligonucleotide aCGH technique. Genome-wide screening using microarrays showed large genomic heterogeneity in MM cases and revealed copy number alterations in $100 \%$ (91/91) of samples. Graphical overview of incidence of genomic CNAs is shown in Figure 1. Overall, we found 1557 CNAs (778 gains and 779 areas of loss of genetic material); median was 16 CNAs per patient (range 1-52). The average size of aberration was 26.2 Mbp; 13\% (204/1557) of all aberrations were smaller than $1 \mathrm{Mbp}$. Detailed description of CNAs found in our cohort is available in Supplementary Table 1 (see Supplementary Material available online at http://dx.doi.org/10.1155/2014/209670).

Our results confirm that there are two distinct wholegenome profiles reflecting major genetic subtypes in MM. Incidence of the extra copies of odd-numbered chromosomes is a common feature of hyperdiploid subgroup, which was found in $50.5 \%(46 / 91)$ of samples, whereas $49.5 \%(45 / 91)$ 


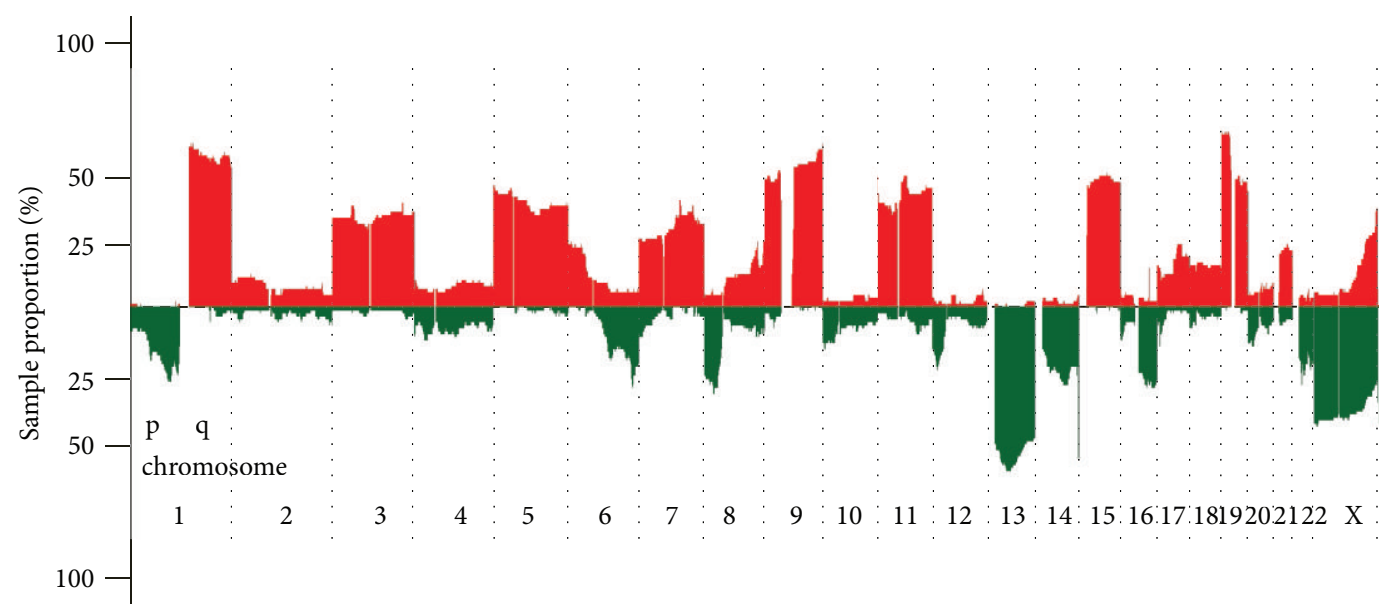

FIGURE 1: Graphical summary of copy number abnormalities in cohort of 91 multiple myeloma patients. Green color represents areas of loss; red corresponds with areas of gain of genetic material.

of cases were nonhyperdiploid. Most often duplicated chromosomes were chromosomes 9 and 15 (both 41.8\%; 38/91), followed by chromosomes 9 (40.7\%; 37/91), 19 (36.3\%; 33/91), 5 (33.0\%; 30/91), 11 (31.9\%; 29/91), 3 (25.3\%; 23/91), 7 (22.0\%; 20/91), and 21 (18.7\%; 17/91).

\subsubsection{CNAs with Prognostic Significance in MM Diagnosis Detected by aCGH}

Deletions in $1 p$. In our study, we found deletion of $1 p$ in $46.2 \%$ of samples (42/91). The whole $1 p$ arm was deleted in $16.7 \%$ (7/42) of cases. Furthermore, we identified three frequently deleted areas of $1 \mathrm{p}$.

Most common deleted locus was located in 1p22.1, where we found $480 \mathrm{Kbp}$ minimal deleted region (MDR), which occurred in $32.9 \%$ of cases (30/91) and included 5 genes (HSP90B3P, TGFER3, BRDT, EPHAX4, and BTBD8). The second frequently deleted region was found in $1 \mathrm{p} 32.3$ band, where we observed deletion in Fas +associated factor 1 (FAF1) and CDKN2C (p18) loci in $19.8 \%$ of cases (18/91). The third $350 \mathrm{Kbp}$ MDR was detected in 1 p12 in 9.9\% (9/91), including loci with MAN1A2, FAM46C, and GDAP2.

Gain 1q. Regions of gain of genetic material in chromosome 1q were found in $71.4 \%(65 / 91)$ of cases; gain of whole $1 \mathrm{q}$ arm was detected in 50.6\% (46/91) of patients. In 5.5\% (5/91) of samples, we defined $10.9 \mathrm{Mbp}$ minimal region of gain (MRG) in 1q21.2-1q23 including CKS1B and ANP32E, two genes associated with poor prognosis in MM.

Chromosome 17. The deletion in $17 \mathrm{p} 13$ locus was found in $14.3 \%$ (13/91). We found MDR of $133.5 \mathrm{Kbp}$ covering 4 genes: ATP1B2, TP53, WRAP5, and EFNB3. Moreover, we found partial gain in $17 \mathrm{q}$ affecting area between $17 \mathrm{q} 21.33$ and $17 \mathrm{qter}$ in $12.1 \%(11 / 91)$, and in 5 cases $(5.5 \%)$ we observed incidence of trisomy 17.

\subsubsection{CNAs Associated with Chromosomes Involved in IgH Rearrangements}

Chromosome 4. The loss of genetic material was frequently observed in $4 \mathrm{p}$. The most common MDR was detected in $4 \mathrm{p} 16.3$ area in $6.6 \%(6 / 91)$, affecting loci of FGFR3 and WHSC1. Furthermore, we found breakpoint in 4 p16.3 locus in 3 cases, resulting probably from unbalanced translocation $\mathrm{t}(4 ; 14)(\mathrm{p} 16 ; \mathrm{q} 32)$. In $7.7 \%$ of cases (7/91), we defined 7.1 Mbp MDR in 4p15.2, comprising twelve genes (LGI2, SEPSECS, PI4K2B, ZCCHC4, ANAPC4, SLC34A2, KIAA0746, LOC389203, RBPJ, CCKAR, TBC1D19, and STIM2).

Chromosome 8. Most common aberration in chromosome 8 was loss of whole $8 \mathrm{p}$, which was found in $23.1 \%$ (21/91) of cases. Aberrationsin 8p24.2 affecting MYC oncogene were found in $31.9 \%(29 / 91)$ of samples, including both gains and deletions $(22 \%, 20 / 91 ; 9.9 \%, 9 / 91$, resp.). In $7.7 \%$ (7/91), we observed breakpoint in the MYC locus, resulting most probably from unbalanced $\mathrm{t}(8 ; 14)$ translocation.

Chromosome 11. We found an extra copy of chromosome 11 in $32.9 \%$ of cases $(30 / 91)$, exclusively in the H-MM group $(P<0.001)$. We identified breakpoint in the CCND1 locus in 4 cases suggesting incidence of $t(11 ; 14)$. Most often deleted region on chromosome 11 was $11 \mathrm{q} 22$ area. We found $4.3 \mathrm{Mbp}$ MDR consisting of 22 genes in 9.9\% (9/91) of cases, including loci of two genes with known function in apoptosis, and connected with the NF- $\kappa \mathrm{B}$ pathway, BIRC2 and BIRC3, and matrix metallopeptidase cluster.

Chromosome 14. The most common aberration was monosomy 14 , which was observed in $17.6 \%$ (16/91) solely in nonhyperdiploid cases $(P<0.001)$. Common MDR was observed in 14q23 region between AKAP5 and ADAM21 genes in $8.8 \%$ $(8 / 91)$ of cases. Another MDR was located in $14 q 32.22$ and 


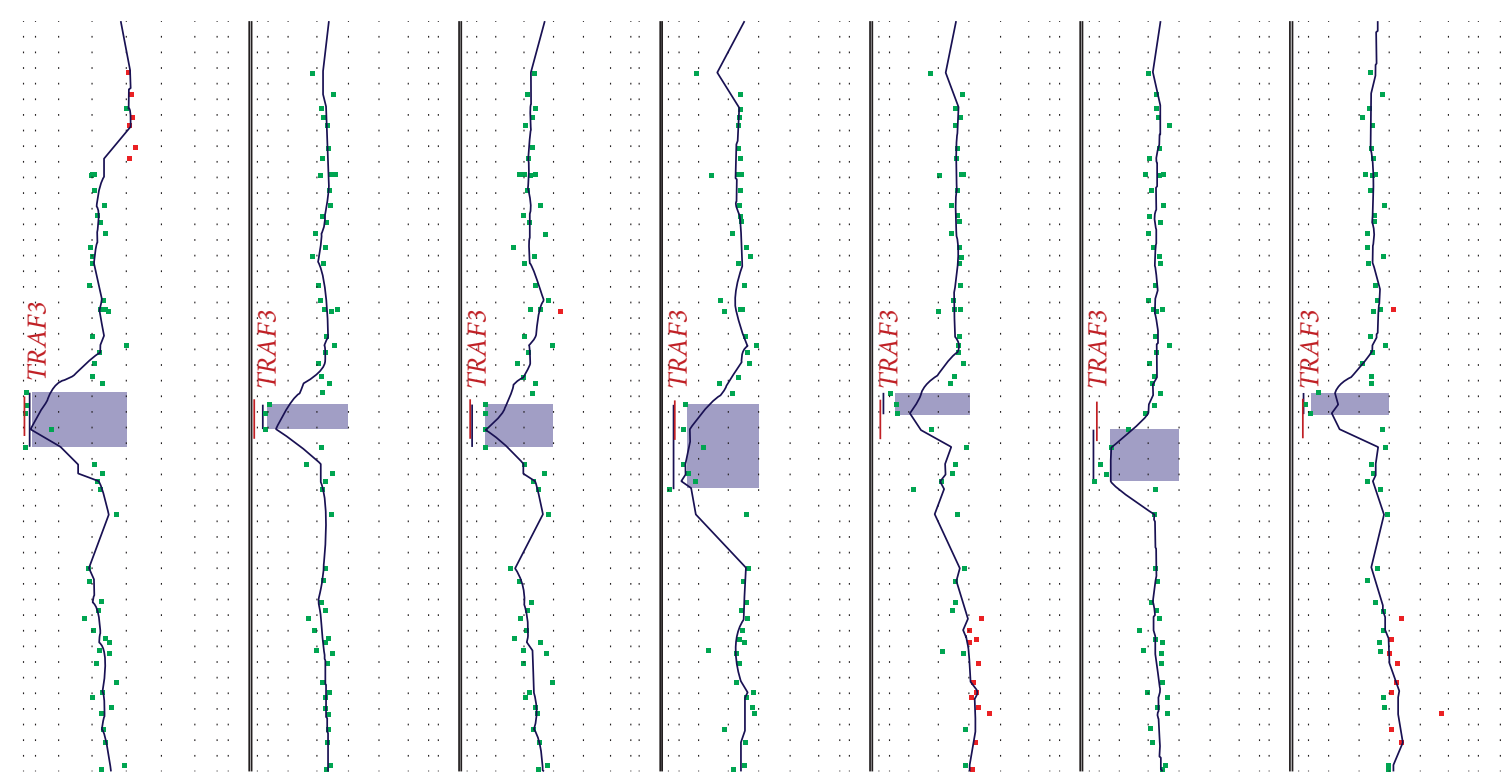

FIGURE 2: Schematic visualization of homozygous deletions in 14q32.33 region with highlighted TRAF3 as main target of deletion in this area.

included 3 genes, TRAF3, $A M N$, and $C D C 42 B P B$. This region was homozygously deleted in incoherent manner (Figure 2) and occurred in $7.7 \%$ (7/91) of cases, with size of deletion varying from $48.7 \mathrm{Kbp}$ to $261 \mathrm{Kbp}$.

Chromosome 16. The loss of $16 \mathrm{q}$ was the most frequent CNA in our cohort of patients $(25.3 \%, 23 / 91)$. In one case with positive $\mathrm{t}(14 ; 16)(\mathrm{q} 32 ; \mathrm{q} 23)$, we found interstitial deletion in fragile site FRA16D including WWOX. In the short arm of chromosome 16 , we found $680 \mathrm{Kbp}$ MDR in 16p13.3 area between CLUAP1 and NAT15 in $7.7 \%$ (7/91).

\subsubsection{Other Regions of Recurrent CNAs}

Chromosome 6. Whereas gains were typical genetic abnormalities for $6 \mathrm{p}$, interstitial deletions were commonly observed in 6q. The most frequently deleted area was 6q25. We defined MDR of size $2.1 \mathrm{Mbp}$, which was observed in $15.4 \%$ (14/91) and was comprised of OPRM1, IPCEF1, CNKSR3, RBM16, TIAM2, TFB1M, CLDN20, and NOX3. Another region with frequent deletion was $6 \mathrm{q} 16.3$, which was found in $11 \%(10 / 91)$ where we defined 3.4 Mbp MDR between COQR and GRIK2. Partial deletion of $6 \mathrm{q}(>75 \%)$ was also observed in $11.0 \%$ $(10 / 91)$ of cases. In $6 \mathrm{p}$, the most common CNA was gain in 6 pter-6p22.3, observed in $8.7 \%$ (9/91).

Chromosome 12. The most common CNA in chromosome 12 was $69.2 \mathrm{Kbp}$ deletion in 12p13. In $12.3 \%$ (13/91) of cases, we found small MDR in 12p13.1 locus including cyclindependent kinase inhibitor $1 \mathrm{~B}(C D K N 1 B)$ and an endothelial cell early response protein gene $A P O L D 1$. In 5 cases, we observed deletion of whole $12 \mathrm{p}$ and 3 cases were missing the whole chromosome 12.

Chromosome 13. The loss of genetic material in chromosome 13 was the most common chromosomal aberration observed in our cohort of patients. The monosomy 13 was found in $50.6 \%(46 / 91)$ of cases. In $8.8 \%(8 / 91)$ of cases, we observed 11.2 Mbp MDR between LRCH1 and DIAHP3 spanning from $13 q 14.2$ to $13 q 21.2$ and containing genes with important roles in cancerogenesis, RB1, DLEU7, and miRNA genes $m i R$ $15 a / m i R-16-1$.

Chromosome 20. The most common aberration observed in chromosome 20 was deletion of the short arm. We found loss affecting approximately $2 / 3$ in $20 \mathrm{p}$ in $8.8 \%$ (8/91) of cases spanning from 20pter to 20p11.23. Gains or losses of whole chromosome 20 were observed in 7.7\% (7/91) of samples ( 4 cases with loss and 3 cases with gain of extra copy of chromosome 20).

Chromosome 22. The most frequent CNA in chromosome 22 was loss of the whole chromosome, which was found in $15.4 \%$ (14/91) of cases. In addition, 6.6\% (6/91) of cases had 33.6 Mbp deletion affecting approximately $1 / 3$ of the $22 \mathrm{q}$ arm between centromere and 22q12.2 band.

3.2.4. Regions with Homozygous Deletions Detected by aCGH. The homozygous deletions (HZDs) play an important role in cancer biology and are considered important genetic aberrations as they are able to fully inactivate genes contained within them. In our cohort of patients, HZDs were found in $30.8 \%$ (28/91) of cases. Median size was $193 \mathrm{Kbs}$ (range $0.039-1.4 \mathrm{Mbp}$ ), and its incidence was noted more often 
in nonhyperdiploid cases $(P=0.02)$. Incidence of most common HZDs is summarized in Table 2. The most frequently affected region was $14 \mathrm{q} 32.32$ with HZD varying from $48.7 \mathrm{Kbp}$ to $261 \mathrm{Kbp}$ observed in $7.7 \%$ (7/91) of cases spanning loci of genes (RCOR1, TRAF3, AMN, and CDC42BPB) in an incoherent manner. The second most common locus with HZD was 1 p32.3 carrying FAF1 and CDKN2C, which was deleted in 5.5\% (5/91) of cases. The HZDs in chromosome 13 were also frequently observed. The HZDs were observed in $6.6 \%(6 / 91)$ of cases, but 13q14.2 locus was hit only in 3 cases (3.3\%), varying from $52 \mathrm{Kbp}$ to $206 \mathrm{Kbp}$ comprising $R B 1$, LPAR6, and RCBTB2. Notably, all single case HZDs affected tumor suppressor genes, such as BRCA2 (13q12.3), INTS6 (13q14.3), or SPRY2 (13q31.1). Further loci with occurrence of HZDs were found in a single case in 1p32.3 (PTPN14; ESRRG), 3q26.3 (PIK3CA), 4q22 (SMARCAD1), 5q15 (MCTP1), 6q22.1 (KPNA5), 11q23.2 (FAM55B, CADM1), 12p31.2 (CDKN1B), 16q23 (WWOX, MAF), and Xq23 (LHFPL1, AMOT).

\subsection{Concordance of Unbalanced Chromosomal Aberrations} Detected by FISH and aCGH. In order to evaluate the possibility of replacing FISH analyses by aCGH in clinical diagnostics, the occurrence of characteristic recurrent unbalanced chromosomal aberrations was tested. The McNemar test was used for comparison of detection of hyperdiploidy, $\operatorname{del}(13)(q 14)$, del (17p13), and gain(1)(q21) detected by I-FISH with the incidence of CNAs in those loci from our aCGH aberration list in $91 \mathrm{MM}$ samples.

In our dataset, a total of $36.3 \%(36 / 91)$ of cases were discordant; however, we did not find statistically significant difference between results from both techniques for single aberrations. Detailed overview is shown in Table 3. While in detection of structural aberrations, concordance was over $90 \%$, the most common discrepancy was observed in detection of the ploidy status $(14.3 \% ; 13 / 91)$. From H-MM cohort evaluated by FISH, 13.3\% (6/45) of cases were classified as nonhyperdiploid by aCGH. Similarly, $15.2 \%$ (7/46) of NHMM samples analyzed by FISH were classified as hyperdiploid by aCGH because of the incidence of extra copies of chromosomes undetectable by FISH multicolor panel. Discordant findings were also found in detection of structural aberrations by both techniques. Of the 91 patients with both aCGH and FISH results, aCGH detected $11.0 \%$ (10/91) of CNAs that were not detected by FISH [ 6 cases of $\operatorname{del}(13)(\mathrm{q} 14)$, 2 cases of $\operatorname{del}(17)(\mathrm{p} 13)$, and 2 cases of $\operatorname{gain}(1)(\mathrm{q} 21)]$. On the contrary, I-FISH detected $11 \%$ (10/91) of abnormalities not identified by aCGH [ 2 cases of $\operatorname{del}(13)(q 14), 3$ cases of $\operatorname{del}(17)(\mathrm{p} 13)$, and 5 cases of gain(1)(q21), resp.].

\section{Discussion}

Detection of chromosomal abnormalities is one of the most important independent prognostic markers in MM pathogenesis and prognosis for patients. Similarly to many other types of hematologic malignancies, MM is characterized by numerous structural and numerical genetic lesions involving many oncogenes, tumor suppressor genes or genes involved in signaling pathways important for cell cycle, apoptosis, and so forth. [2]. While karyotyping techniques are able to detect chromosomal abnormalities roughly in $30 \%$ of cells because of the low proliferative activity, the introduction of new cytogenetic techniques, such as I-FISH or aCGH, allows us to detect genetic lesions in more than $90 \%$ of malignant PCs [18]. Expanded panel of FISH markers includes $\operatorname{del}(13)(\mathrm{q} 14) /$ monosomy $13, \mathrm{t}(11 ; 14)(\mathrm{q} 13 ; \mathrm{q} 32)$, $\mathrm{t}(14 ; 16)(\mathrm{q} 32 ; \mathrm{p} 23)$, and hyperdiploidy. Even though I-FISH is nowadays considered as a gold standard for cytogenetic investigations in MM, it may be insufficient for description of given genetic heterogeneity. Moreover, several studies proved virtually $100 \%$ occurrence of CNAs in MM when techniques of whole-genome screening were used in MM diagnosis [19]. Based on the above-mentioned studies, we utilized the global assessment of genomic abnormalities via a high-resolution Agilent $4 \mathrm{x} 44 \mathrm{k}$ aCGH platform and in combination with FISH in $91 \mathrm{MM}$ patients. To the best of our knowledge, this is the first study of such scale in central Europe in MM patients.

4.1. FISH Assessment of Cytogenetic Aberrations Wit Prognostic Significance. Rearrangements of the $\mathrm{IgH}$ locus play important role in MM pathogenesis. Several studies showed negative prognostic impact of $\mathrm{t}(4 ; 14)(\mathrm{p} 16 ; \mathrm{q} 32)$ in newly diagnosed or relapsed patients. In our cohort, we found $\mathrm{t}(4 ; 14)$ (p16;q32) using FISH in $17.4 \%(15 / 86)$ of cases in agreement with previous reports [20]. The $\operatorname{del}(17)(\mathrm{p} 13)$ including tumor suppressor gene TP53 is considered an important negative prognostic factor in $\mathrm{MM}$ pathogenesis. In our cohort, we found $\operatorname{del}(17)(\mathrm{p} 13)$ in $15.4 \%$ (14/91) of cases in concordance with previous observations [21]. Gain(1)(q21) and subsequently overexpression of $C K S 1 B$ are nowadays considered as an independent prognostic factor in $\mathrm{MM}$ diagnosis. In our previous studies, we showed that incidence of this genetic lesion is associated with poor prognosis when detected by I-FISH in both newly and relapsed MM patients. In this cohort, we found gain(1)(q21) in 53\% of cases, which is in agreement with previously published results [22].

4.2. Whole-Genome Screening by Oligo-Based Microarrays and aCGH Technique. The whole-genome screening using aCGH identified CNAs $>100 \mathrm{Kbp}$ in $100 \%$ of cases. Most common CNAs were found in 1p, 1q, 6p, 8p, 13q, 14q, 16q, and $22 \mathrm{q}$ along with gain of extra copies of odd-numbered chromosomes. Hyperdiploidy was found in nearly half of the cases $(47.3 \% ; 43 / 91)$. Within the hyperdiploid cohort of patients, we observed incidence of trisomy 11 as well as gain(1q) and $\operatorname{del}(13 q)$ associated with worse prognosis; however, the association did not meet statistical significance $(P=0.112)$, as previously described [23].

In addition to current high-risk panel genetic abnormalities, several other CNAs associated with adverse prognosis were recently identified by genome-wide techniques. In chromosome 1, deletion in 1p32 affecting loci of CDKN2C and FAF1 is connected with shorter OS. In our cohort, we defined MDR in 1p32.3 locus with incidence of this focal deletion in $19.8 \%$ (18/91), which is in good agreement with previous observations [24]. Another frequent deletion in $1 p$ was found in 1p12, including loci of MAN1A2, GDAP2, and 
TABLE 2: Incidence of most common homozygous deletion in a cohort of 91 multiple myeloma patients detected by array-CGH technique.

\begin{tabular}{lccc}
\hline Chromosome location & Size $(\mathrm{Mb})$ & Prevalence $(\%)$ & Genes \\
\hline $14 \mathrm{q} 32.32$ & $0.063-0.261$ & 7.7 & RCOR1, TRAF3, AMN, CDC42BPB \\
$1 \mathrm{p} 32.3$ & $0.068-0.387$ & 5.5 & FAF1, CDKN2C \\
$11 \mathrm{q} 22.1-11 \mathrm{q} 22.3$ & $3.6-4.7$ & 2.8 & BIRC3, BIRC2, MMP cluster \\
$13 \mathrm{q} 14.2$ & $0.053-0.206$ & 2.8 & RB1, P2RY5, RCBTB2 \\
$16 \mathrm{q} 12.1-16 \mathrm{q} 12.2$ & $1.40-1.42$ & 1.9 & CYLD, SALL1 \\
\hline
\end{tabular}

TABLE 3: Comparison of array-CGH and FISH results in evaluation of cytogenetic aberrations with known effect on prognosis in multiple myeloma patients.

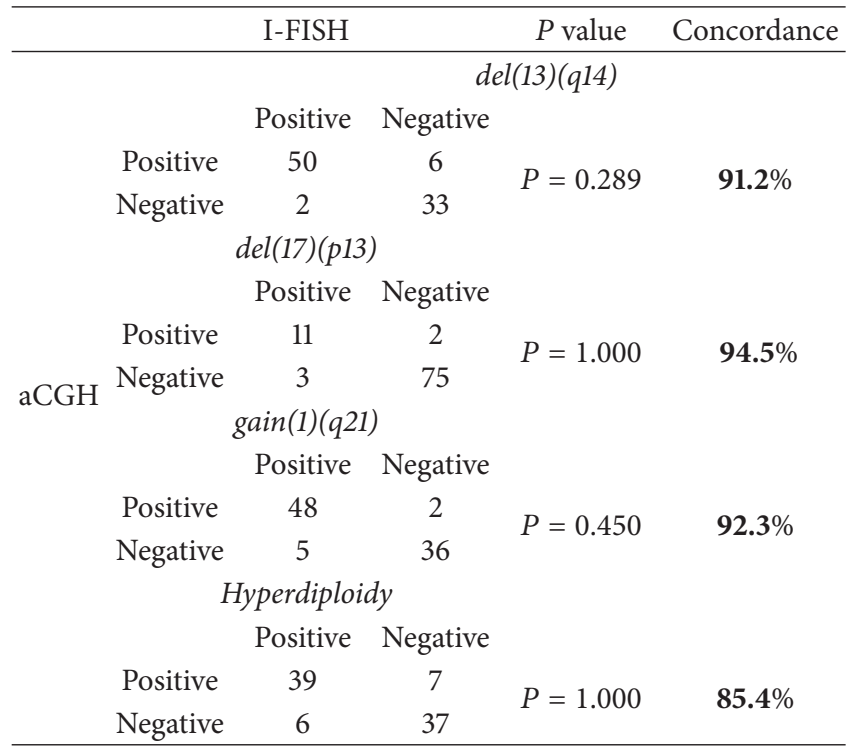

FAM46C. Recently, incidence of mutations and deletion of FAM46C were described and associated with impaired OS in MM patients [25]. In 1q, we observed common region of gain of genetic sequences in 1q21.2 region with two genes associated with negative impact on prognosis, $C K S 1 B$, and ANP32E [26]. In agreement with previous studies, our results also showed that in most cases, the whole 1q arm is affected [27]. Genetic lesions involving deletions of TP53 in $17 \mathrm{p} 13.1$ were observed in $13.2 \%$ (12/91). Even though there is agreement about loss/mutation of TP53 having negative impact on MM prognosis, MDR in 17p13.1 area in our dataset also included spermine N1-acetyltransferase SAT2, which has been reported to be significantly underexpressed in $\operatorname{del}(17 p)$; it interacts with p65 subunit of the NF- $\kappa$ B pathway and thus is another possible candidate gene in this area [28].

\subsection{Incidence and Impact of Homozygous Deletions.} Homozygous deletions play important role in cancer biology and are considered to be important genetic events. By the definition, this event is able to fully inactivate genes contained within them. The most frequent region associated with incidence of HZD in our dataset was 14q32 (7.7\%; $7 / 91)$. The incidence of del(14q) is commonly observed in hematological malignancies. In MM, several studies recently showed that TNF receptor-associated factor 3 (TRAF3) is an important target of deletion in this locus. TRAF3 is associated with negative induction of noncanonical NF- $\kappa$ B pathway, enhances BIRC2/BIRC3 mediated proteasome degradation of NF- $\kappa \mathrm{B}$ inducing kinase (NIK), and thus increases autonomy of tumor PCs from the bone marrow microenvironment [29]. Similarly to others, TRAF3 was the target of HZDs in our MDR in 14q32.33; its incidence was comparable with other MM studies as well as other B-lymphomas [30, 31]. Another region affected with HZD was 1q32.3 carrying loci of FAF1 and CDKN2C, which occurred in 5.5\% (5/91) of cases. Incidence of HZD in this region is associated with adverse prognosis in MM patients and is also common in mantle cell lymphoma patients [32]. Finally, HZD regions observed in $<5 \%$ of cases have relevance in MM biology due to involvement in important signaling pathways, such as NF- $\kappa \mathrm{B}$ (CYLD, BIRC2, and BIRC3), regulation of cell cycle $(C D K N 1 B, R B 1)$, or connection with apoptosis (WWOX, FAF1) $[33,34]$.

4.4. Comparison of FISH Evaluation and aCGH Results in Cohort of $91 \mathrm{MM}$ Patients. Molecular cytogenetic analysis using I-FISH technique is still considered to be a golden standard for cytogenetic evaluation in MM diagnosis. However, genomic profiling using aCGH provides information beyond the commonly detected unbalanced genetic lesions that are observed by FISH. In our cohort, chromosomal aberrations were detected in $93.4 \%$ (85/91) of cases using I-FISH, while aCGH screening was able to detect CNAs in $100 \%$ (91/91) of cases. The concordance for loss of $R B 1$ (13q14), TP53 (17p13), gain(1)(q21), and hyperdiploidy was $91.2 \%, 94.5 \%, 92.3 \%$, and $85.4 \%$, respectively; median of concordance for all aberrations was $91.8 \%$. To our knowledge, no similar study was done in MM, but there are data from different hematological malignancies. Comparative studies in chronic myeloid leukemia (CLL) between FISH and aCGH showed high degree of concordance with our results, with the concordance between FISH and aCGH reaching up to $93 \%$ and $95.5 \%$, respectively $[35,36]$. Proportion of structural abnormalities missed by FISH and aCGH was $18.7 \%$ (17/91) and $17.6 \%(16 / 91)$, respectively, which is a little higher than in previous studies; however, we used a larger cohort of patients and highly purified CD138+ sorted cells as starting material instead of bone marrow samples, which could affect specificity of our analysis over previously published data showing discrepancy from 9 to $12 \%[37,38]$. In addition, several other studies in MM and CLL also showed that aCGH 
is less effective when incidence of CNAs is presented to be $<30 \%$ of the cells $[30,35,39]$. In our dataset, $37.5 \%(6 / 16)$ of cases with missed CNAs by aCGH fell within this condition (2x loss of $R B 1,2 \mathrm{x}$ gain $C K S 1 B$, and $3 \mathrm{x}$ loss of TP53). These cases are hard to evaluate by default setting of the analytic software; however, novel computing algorithms developed for detection of mosaic samples are able to overcome this issue [40]. Primary reason for discrepancy in detection of hyperdiploidy was caused by higher false positivity of FISH evaluations, when FISH signals were scored as trisomies when only a part of the chromosome arm was duplicated but not the whole chromosome.

\section{Conclusions}

The results of our study showed that our complex approach comprising cell sorting, I-FISH evaluation of balanced chromosomal changes (IgH rearrangement and translocations associated with adverse prognosis for MM patients), and genome-wide profiling gives us a robust diagnostic tool suitable for precise evaluation of the high-risk genetic lesions. The utilization of whole-genome CGH microarrays is able to substitute routine FISH evaluations in detection of unbalanced genetic lesions with prognostic impact in MM and bring additional information about changes in genome of malignant plasma cells even though the detection of clonal aberrations in MM samples could be challenging. Altogether, combination of aCGH and I-FISH technique gives us new opportunities for description of genetic heterogeneity in MM and thus identification of novel cytogenetic features capable of discerning prognosis in MM. However, further studies focusing on genetic background of MM are needed for better understanding and characterization of role of genetic changes in MM pathology.

\section{Conflict of Interests}

All authors have no conflict of interests (including any financial relationship with companies/products) regarding the publication of this paper.

\section{Acknowledgments}

The authors would like to thank all participating members of the Czech Myeloma Group, especially all patients and their caregivers for making this work possible. The research leading to these results has received funding from the European Union Seventh Framework Programme (FP7/20072013) under Grant Agreement no. 278570 and OP VK CZ.1.07/2.3.00/20.0183 Project. This study was supported by research Grant MSM0021622434 from the Ministry of Education, Youth and Sports, Czech Republic, and by Grant nos. NT11154, NT13190, NT12130, and NT13492 from the Internal Grant Agency of the Ministry of Health of the Czech Republic. This research was supported by Ministry of Health, Czech Republic, for conceptual development of research organization (FNBr 65269705). The authors would also like to thank John B. Smith for proofreading the paper.

\section{References}

[1] M. Dimopoulos, R. Kyle, J.-P. Fermand et al., "Consensus recommendations for standard investigative workup: report of the International Myeloma Workshop Consensus Panel 3," Blood, vol. 117, no. 18, pp. 4701-4705, 2011.

[2] R. Fonseca, B. Barlogie, R. Bataille et al., "Genetics and cytogenetics of multiple myeloma: a workshop report," Cancer Research, vol. 64, no. 4, pp. 1546-1558, 2004.

[3] R. Fonseca, D. Harrington, M. M. Oken et al., "Biological and prognostic significance of interphase fluorescence in situ hybridization detection of chromosome 13 abnormalities $(\Delta 13)$ in multiple myeloma: an Eastern Cooperative Oncology Group study," Cancer Research, vol. 62, no. 3, pp. 715-720, 2002.

[4] N. V. Smadja, C. Bastard, C. Brigaudeau, D. Leroux, and C. Fruchart, "Hypodiploidy is a major prognostic factor in multiple myeloma," Blood, vol. 98, no. 7, pp. 2229-2238, 2001.

[5] J. Shaughnessy, "Amplification and overexpression of CKS1B at chromosome band 1q21 is associated with reduced levels of p27 Kip1 and an aggressive clinical course in multiple myeloma," Hematology, vol. 10, no. 1, pp. 117-126, 2005.

[6] R. Fonseca, S. A. Van Wier, W. J. Chng et al., "Prognostic value of chromosome 1q21 gain by fluorescent in situ hybridization and increase CKS1B expression in myeloma," Leukemia, vol. 20, no. 11, pp. 2034-2040, 2006.

[7] N. C. Gutiérrez, J. L. García, J. M. Hernández et al., "Prognostic and biologic significance of chromosomal imbalances assessed by comparative genomic hybridization in multiple myeloma," Blood, vol. 104, no. 9, pp. 2661-2666, 2004.

[8] B. A. Walker and G. J. Morgan, "Use of single nucleotide polymorphism-based mapping arrays to detect copy number changes and loss of heterozygosity in multiple myeloma," Clinical Lymphoma and Myeloma, vol. 7, no. 3, pp. 186-191, 2006.

[9] C. Largo, S. Alvarez, B. Saez et al., "Identification of overexpressed genes in frequently gained/amplified chromosome regions in multiple myeloma," Haematologica, vol. 91, no. 2, pp. 184-191, 2006.

[10] M. A. Chapman, M. S. Lawrence, J. J. Keats et al., "Initial genome sequencing and analysis of multiple myeloma," Nature, vol. 471, no. 7339, pp. 467-472, 2011.

[11] P. Nemec, Z. Zemanova, P. Kuglik et al., "Complex karyotype and translocation $\mathrm{t}(4 ; 14)$ define patients with high-risk newly diagnosed multiple myeloma: results of CMG2002 trial," Leukemia and Lymphoma, vol. 53, no. 5, pp. 920-927, 2012.

[12] H. Greslikova, R. Zaoralova, H. Filkova et al., "Negative prognostic significance of two or more cytogenetic abnormalities in multiple myeloma patients treated with autologous stem cell transplantation," Neoplasma, vol. 57, no. 2, pp. 111-117, 2010.

[13] J. Cumova, L. Kovarova, A. Potacova et al., "Optimization of immunomagnetic selection of myeloma cells from bone marrow using magnetic activated cell sorting," International Journal of Hematology, vol. 92, no. 2, pp. 314-319, 2010.

[14] J. Smetana, J. Fröhlich, V. Vranová, A. Mikulášová, P. Kuglík, and R. Hájek, "Oligonucleotide-based array CGH as a diagnostic tool in multiple myeloma patients," Klinicka Onkologie, vol. 24, pp. S43-S48, 2011.

[15] D. Lipson, Y. Aumann, A. Ben-Dor, N. Linial, and Z. Yakhini, "Efficient calculation of interval scores for DNA copy number data analysis," Journal of Computational Biology, vol. 13, no. 2, pp. 215-228, 2006. 
[16] G. J. Ahmann, S. M. Jalal, A. L. Juneau et al., "A novel threecolor, clone-specific fluorescence in situ hybridization procedure for monoclonal gammopathies," Cancer Genetics and Cytogenetics, vol. 101, no. 1, pp. 7-11, 1998.

[17] F. M. Ross, H. Avet-Loiseau, G. Ameye et al., "Report from the European myeloma network on interphase FISH in multiple myeloma and related disorders," Haematologica, vol. 97, no. 8, pp. 1272-1277, 2012.

[18] S. Kumar, R. Fonseca, R. P. Ketterling et al., "Trisomies in multiple myeloma: impact on survival in patients with high-risk cytogenetics," Blood, vol. 119, no. 9, pp. 2100-2105, 2012.

[19] R. Fonseca, P. L. Bergsagel, J. Drach et al., "International Myeloma Working Group molecular classification of multiple myeloma: spotlight review," Leukemia, vol. 23, no. 12, pp. 22102221, 2009.

[20] N. C. Gutiérrez, M. V. Castellanos, M. L. Martín et al., "Prognostic and biological implications of genetic abnormalities in multiple myeloma undergoing autologous stem cell transplantation: $\mathrm{t}(4 ; 14)$ is the most relevant adverse prognostic factor, whereas RB deletion as a unique abnormality is not associated with adverse prognosis," Leukemia, vol. 21, no. 1, pp. 143-150, 2007.

[21] L. Lodé, M. Eveillard, V. Trichet et al., "Mutations in TP53 are exclusively associated with del(17p) in multiple myeloma," Haematologica, vol. 95, no. 11, pp. 1973-1976, 2010.

[22] J. Smetana, K. Berankova, R. Zaoralova et al., "Gain(1)(q21) is an unfavorable genetic prognostic factor for patients with relapsed multiple myeloma treated with thalidomide but not for those treated with bortezomib," Clinical Lymphoma, Myeloma and Leukemia, vol. 13, no. 2, pp. 123-130, 2013.

[23] D. R. Carrasco, G. Tonon, Y. Huang et al., "High-resolution genomic profiles define distinct clinico-pathogenetic subgroups of multiple myeloma patients," Cancer Cell, vol. 9, no. 4, pp. 313325, 2006.

[24] H. Avet-Loiseau, C. Li, F. Magrangeas et al., "Prognostic significance of copy-number alterations in multiple myeloma," Journal of Clinical Oncology, vol. 27, no. 27, pp. 4585-4590, 2009.

[25] K. D. Boyd, F. M. Ross, B. A. Walker et al., "Mapping of chromosome 1p deletions in myeloma identifies FAM46C at $1 \mathrm{p} 12$ and $\mathrm{CDKN} 2 \mathrm{C}$ at $1 \mathrm{p} 32.3$ as being genes in regions associated with adverse survival," Clinical Cancer Research, vol. 17, no. 24, pp. 7776-7784, 2011.

[26] B. A. Walker, P. E. Leone, L. Chiecchio et al., "A compendium of myeloma-associated chromosomal copy number abnormalities and their prognostic value," Blood, vol. 116, no. 15, pp. e56-e65, 2010.

[27] H. Chang, X. Qi, A. Jiang, W. Xu, T. Young, and D. Reece, "1p21 deletions are strongly associated with 1q21 gains and are an independent adverse prognostic factor for the outcome of high-dose chemotherapy in patients with multiple myeloma," Bone Marrow Transplantation, vol. 45, no. 1, pp. 117-121, 2010.

[28] K. D. Boyd, F. M. Ross, W. J. Tapper et al., "The clinical impact and molecular biology of del(17p) in multiple myeloma treated with conventional or thalidomide-based therapy," Genes Chromosomes and Cancer, vol. 50, no. 10, pp. 765-774, 2011.

[29] Y. N. Demchenko, O. K. Glebov, A. Zingone, J. J. Keats, P. Leif Bergsagel, and W. Michael Kuehl, "Classical and/or alternative NF- $\kappa$ B pathway activation in multiple myeloma," Blood, vol. 115, no. 17, pp. 3541-3552, 2010.

[30] J. J. Keats, R. Fonseca, M. Chesi et al., "Promiscuous mutations activate the noncanonical NF- $\kappa \mathrm{B}$ pathway in multiple myeloma," Cancer Cell, vol. 12, no. 2, pp. 131-144, 2007.
[31] E. Braggio, J. J. Keats, X. Leleu et al., "Identification of copy number abnormalities and inactivating mutations in two negative regulators of nuclear factor- $\kappa \mathrm{B}$ signaling pathways in Waldenström's macroglobulinemia," Cancer Research, vol. 69, no. 8, pp. 3579-3588, 2009.

[32] N. J. Dickens, B. A. Walker, P. E. Leone et al., "Homozygous deletion mapping in myeloma samples identifies genes and an expression signature relevant to pathogenesis and outcome," Clinical Cancer Research, vol. 16, no. 6, pp. 1856-1864, 2010.

[33] S. Gardam, V. M. Turner, H. Anderton et al., "Deletion of cIAP1 and cIAP2 in murine B lymphocytes constitutively activates cell survival pathways and inactivates the germinal center response," Blood, vol. 117, no. 15, pp. 4041-4051, 2011.

[34] R. Di Fiore, A. D'Anneo, G. Tesoriere, and R. Vento, "RB1 in cancer: different mechanisms of RB1 inactivation and alterations of pRb pathway in tumorigenesis," Journal of Cellular Physiology, vol. 228, no. 8, pp. 1676-1687, 2013.

[35] S. R. Gunn, M. S. Mohammed, M. E. Gorre et al., "Wholegenome scanning by array comparative genomic hybridization as a clinical tool for risk assessment in chronic lymphocytic leukemia," Journal of Molecular Diagnostics, vol. 10, no. 5, pp. 442-451, 2008.

[36] A. Tyybäkinoja, J. Vilpo, and S. Knuutila, "High-resolution oligonucleotide array-CGH pinpoints genes involved in cryptic losses in chronic lymphocytic leukemia," Cytogenetic and Genome Research, vol. 118, no. 1, pp. 8-12, 2007.

[37] A. Patel, S.-H. Kang, P. A. Lennon et al., "Validation of a targeted DNA microarray for the clinical evaluation of recurrent abnormalities in chronic lymphocytic leukemia," American Journal of Hematology, vol. 83, no. 7, pp. 540-546, 2008.

[38] D. P. O'Malley, C. Giudice, A. S. Chang et al., "Comparison of array comparative genomic hybridization (aCGH) to FISH and cytogenetics in prognostic evaluation of chronic lymphocytic leukemia," International Journal of Laboratory Hematology, vol. 33, no. 3, pp. 238-244, 2011.

[39] C. Largo, B. Saéz, S. Alvarez et al., "Multiple myeloma primary cells show a highly rearranged unbalanced genome with amplifications and homozygous deletions irrespective of the presence of immunoglobulin-related chromosome translocations," Haematologica, vol. 92, no. 6, pp. 795-802, 2007.

[40] R. Valli, E. Maserati, C. Marletta, B. Pressato, F. Lo Curto, and F. Pasquali, "Evaluating chromosomal mosaicism by array comparative genomic hybridization in hematological malignancies: the proposal of a formula," Cancer Genetics, vol. 204, no. 4, pp. 216-218, 2011. 


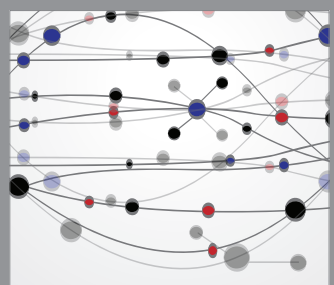

The Scientific World Journal
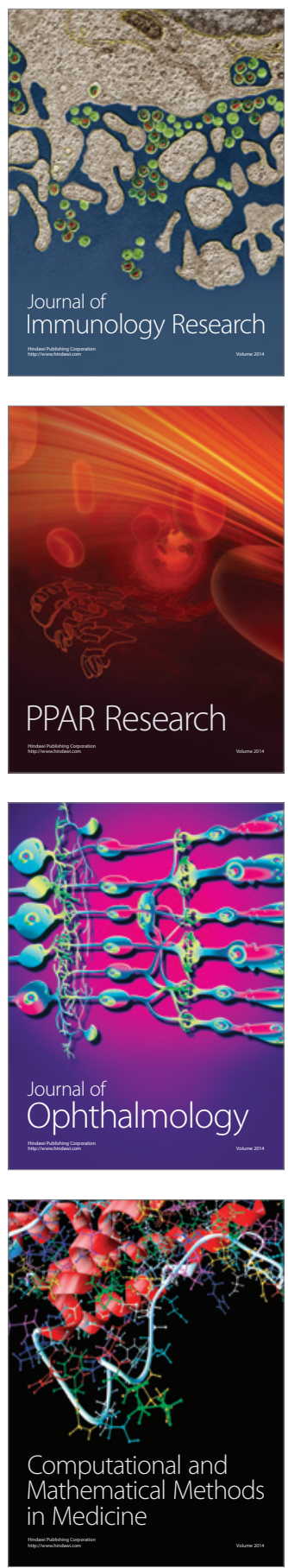

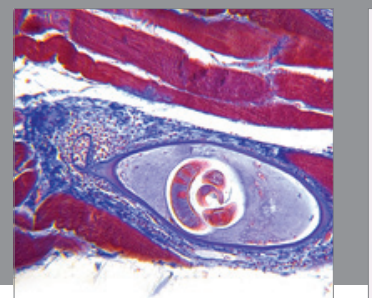

Gastroenterology

Research and Practice
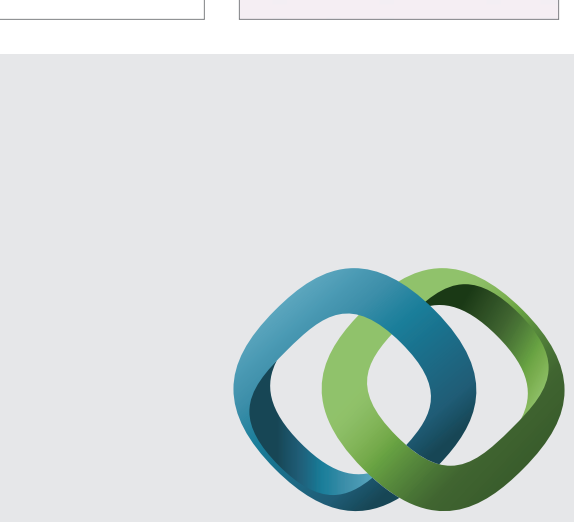

\section{Hindawi}

Submit your manuscripts at

http://www.hindawi.com
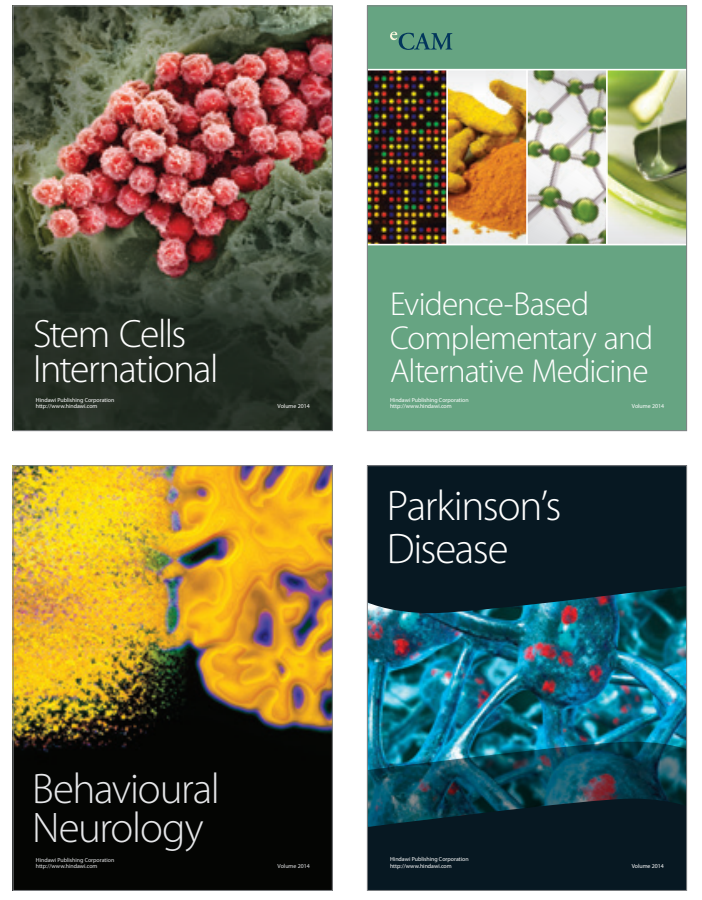
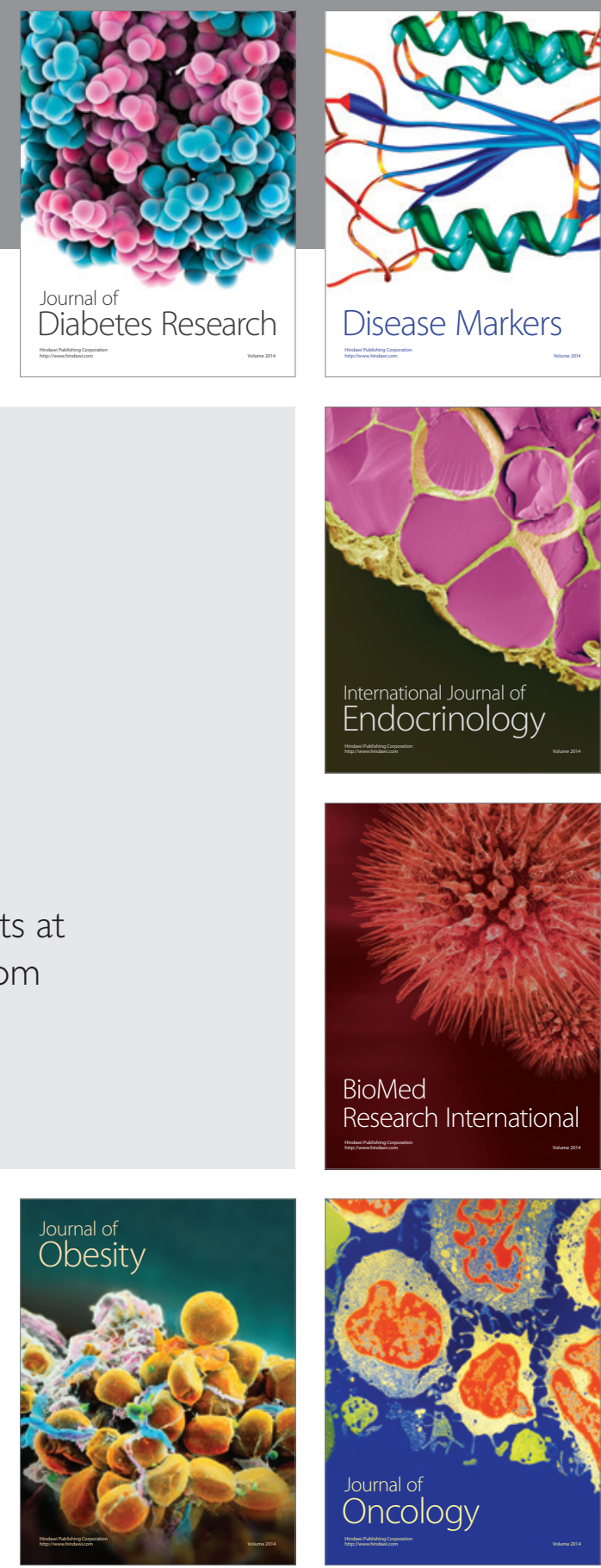

Disease Markers
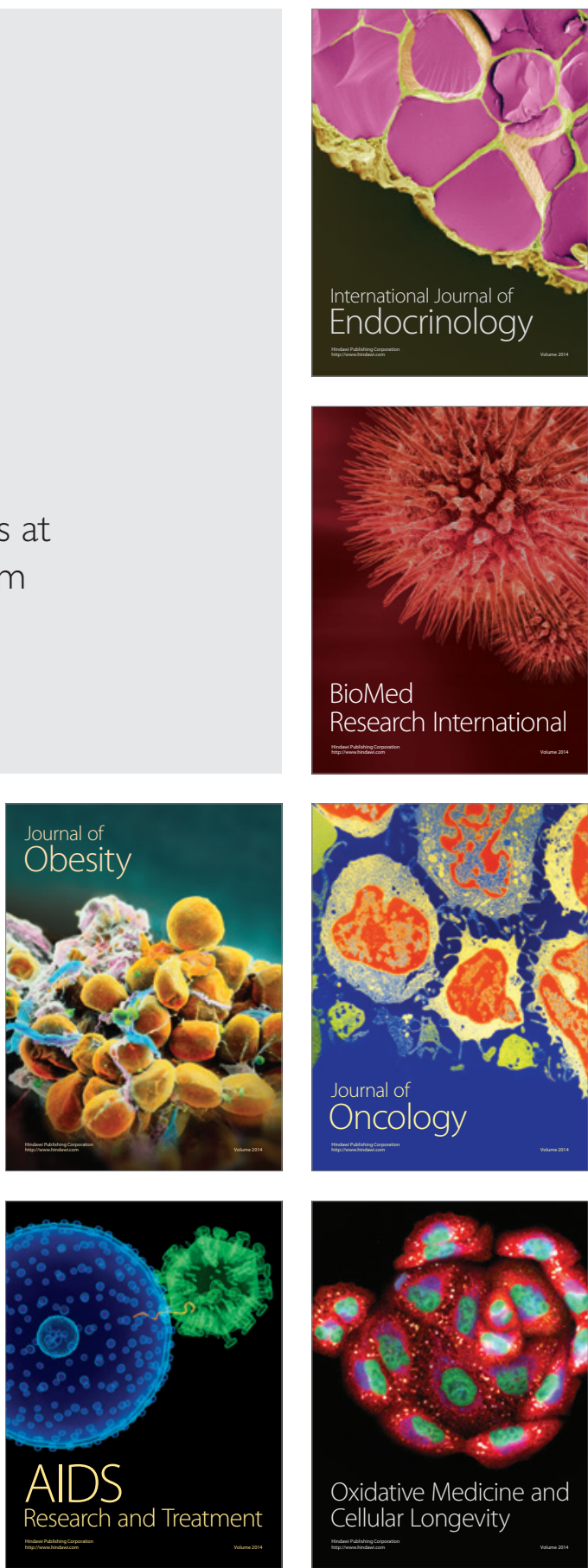